\title{
Tipologia facial aplicada à Fonoaudiologia: revisão de literatura
}

\section{Facial types applied to Speech-Language Pathology: literature review}

\author{
Rossana Ribeiro Ramires ${ }^{1}$, Léslie Piccolotto Ferreira², Irene Queiroz Marchesan³, Débora Martins Cattoni \\ Marta Assumpção de Andrada e Silva ${ }^{5}$
}

\begin{abstract}
RESUMO
A face humana, com suas estruturas ósseas e musculares, apresenta características próprias e peculiares. Pode ser classificada em três tipos básicos, os quais têm relação com a variação do formato e da configuração craniofacial, tanto no sentido vertical como no horizontal e influenciam diretamente a oclusão dentária, harmonia facial, musculatura orofacial e funções estomatognáticas. Por essa razão, diagnosticar o tipo facial é importante para a clínica fonoaudiólogica. O objetivo deste estudo foi realizar uma revisão de literatura relacionada às características dos tipos faciais e apresentar as pesquisas e os estudos mais recentes sobre o tema. Para atingir tal meta, fez-se um levantamento bibliográfico nas bases de dados LILACS, SciELO, Web of Science e Google Acadêmico, além de livros, dissertações e teses sobre o assunto dos últimos dez anos. Várias pesquisas em campo comprovaram algumas características dos tipos faciais encontradas, principalmente, em referências mais antigas. Alguns aspectos, porém, foram controversos ao se comparar os tipos faciais como a atividade eletromiográfica do músculo masseter, modo respiratório e o comprimento do lábio superior e do filtro. Pôde-se constatar que conhecer o tipo facial e correlacioná-lo às funções estomatognáticas, musculatura e oclusão é um fator importante para a prática clínica, mas o profissional deve ser flexível ao comparar as características do paciente com a literatura. Dessa forma, pode-se evitar determinar uma anormalidade ou atipia quando não for o caso, e ocorrer apenas uma variabilidade ou adaptação.
\end{abstract}

Descritores: Face/fisiologia; Sistema estomatognático; Oclusão dentária; Diagnóstico

\section{INTRODUÇÃO}

Tipologia facial é a variação do esqueleto craniofacial, o qual é composto de estruturas ósseas e musculares. Diag-

Trabalho realizado no Programa de Estudos Pós-Graduados em Fonoaudiologia da Pontifícia Universidade Católica de São Paulo - PUC-SP - São Paulo (SP), Brasil, como parte de uma dissertação de mestrado.

(1) Mestre, Integrante do Corpo Técnico Diretor do Grupo de Apoio ao Indivíduo com Autismo - GAIA - São José dos Campos (SP), Brasil.

(2) Doutora, Professora Titular da Graduação e Pós-Graduação em Fonoaudiologia da Pontifícia Universidade Católica de São Paulo - PUCSP - São Paulo (SP), Brasil; Docente do Curso de Especialização em Fonoaudiologia - Voz da Coordenadoria Geral de Especialização, Aperfeiçoamento e Extensão da Pontifícia Universidade Católica de São Paulo - CEGEAE/PUC-SP - São Paulo (SP), Brasil.

(3) Doutora, Diretora clínica do CEFAC Pós-Graduação em Saúde e Educação - São Paulo (SP), Brasil.

(4) Doutora, Professora do CEFAC Pós-Graduação em Saúde e Educação São Paulo (SP), Brasil.

(5) Doutora, Professora Assistente da Graduação e Pós-Graduação em Fonoaudiologia da Pontifícia Universidade Católica de São Paulo - PUC-SP - São Paulo (SP), Brasil; Professora Adjunto do Curso de Fonoaudiologia da Faculdade de Ciências Médicas da Santa Casa de São Paulo - FCMSCSP São Paulo (SP), Brasil; Professora dos Cursos de Especialização em Voz da da Coordenadoria Geral de Especialização, Aperfeiçoamento e Extensão da Pontifícia Universidade Católica de São Paulo - CEGEAE/PUC-SP - São Paulo (SP), Brasil e do CEFAC Pós-Graduação em Saúde e Educação - São Paulo (SP), Brasil.

Endereço para correspondência: Rossana Ribeiro Ramires. R. Teopompo de Vasconcelos, 375/163, Vila Adyana, São José dos Campos (SP), Brasil, CEP: 12243-830. E-mail: rossana_ramires@yahoo.com.br

Recebido em: 8/9/2009; Aceito em: 29/10/2009 nosticar o tipo facial é importante, pois cada tipo apresenta características próprias de acordo com a oclusão dentária, a harmonia facial, a musculatura orofacial, além do formato e da configuração das estruturas craniofaciais. Sabe-se que esses aspectos influenciam diretamente as funções de mastigação, deglutição, voz, respiração e fala.

Por essa razão, determinar o tipo de face dos indivíduos é fundamental para profissionais como ortodontistas, ortopedistas faciais, cirurgiões plásticos e bucomaxilofaciais e fonoaudiólogos, principalmente aqueles que atuam na área de motricidade orofacial.

O objetivo deste estudo foi realizar uma revisão de literatura relacionada às características dos tipos faciais e apresentar as pesquisas e os estudos mais recentes sobre o tema. Para atingir tal meta, fez-se um levantamento bibliográfico nas bases de dados LILACS, SciELO, Web of Science e Google Acadêmico, além de livros, dissertações e teses sobre o assunto publicadas nos últimos 10 anos.

\section{REVISÃO DE LITERATURA}

A tipologia facial constitui a variação das estruturas ósseas e musculares, dentro da normalidade. Tem relação direta com o crescimento e a variação da forma da base óssea orofacial que é composta pelos ossos maxilares, mandíbula, dentes e articulações temporomandibulares ${ }^{(1)}$.

Existem diversas formas de se classificar os tipos faciais. Uma das mais utilizadas mantém relação direta com o 
crescimento craniofacial e divide a face em: dolicofacial, dolicocefálica ou leptoprosopa; mesofacial, mesocefálica ou mesoprosopa; e braquifacial, braquicefálica ou euriprosopa ${ }^{(1-14)}$. Outra terminologia classifica a face como: hiperdivergente, crescimento predominante no sentido horário; neutra, quando ocorre direto para baixo; ou hipodivergente, predominância do sentido anti-horário ${ }^{(9,15)}$. Mais uma classificação que considera o sentido vertical da face a divide em longa, média ou $\operatorname{curta}^{(4-5,16-18)}$.

De acordo com o perfil e a posição da maxila e da mandíbula em relação à base anterior do crânio, a face pode ser: retrognata, retrovertida ou convexa; ortognata, neutrovertida ou reta; ou prognata, provertida ou côncava ${ }^{(2,19)}$. Além disso, o perfil pode ser considerado biprotruso ${ }^{(20)}$.

Existe uma tendência recente de classificação dos tipos faciais, fortemente baseada nas características clínicas de cada face. Na verdade, consiste em uma nova classificação das mal oclusões, que não leva em consideração apenas a relação molar e a posição dentária, mas também o padrão de crescimento craniofacial. Nessa linha, os indivíduos podem apresentar padrão I, II, III, face longa ou face curta; todos os cinco padrões com características próprias. Deve-se avaliar também se a face é considerada agradável, aceitável ou desagradável para se conquistar melhores resultados estéticos durante o tratamento ortodôntico $^{(21)}$.

No que diz respeito às características de cada tipo facial, várias pesquisas apresentadas a seguir e dispostas seguindo a ordem cronológica trouxeram contribuições quanto a diversos aspectos referentes às estruturas craniofaciais e funções estomatognáticas. Ao se realizar o levantamento bibliográfico dos últimos dez anos, foram encontradas 18 referências nos anos de 2001 a 2009.

Em 2001, foi encontrado um artigo no qual se investigou, a partir de uma revisão de literatura, a relação entre voz e tipologia facial. Constatou-se que as pessoas de face longa apresentam qualidade vocal grave com tendência a ser abafada e escura, associada à ressonância posterior. E as pessoas de face curta se caracterizam por qualidade vocal aguda com tendência metálica e ressonância faríngea ${ }^{(22)}$.

Outra revisão de literatura foi realizada em 2002; nesse caso, sobre a dimensão vertical da face mediante ao estudo de vários aspectos que foram associados à face longa, média e curta e/ou mordida aberta e profunda. Dentre as considerações expostas, ressaltou-se que as medidas da altura facial anterior inferior são significativamente diferentes entre os indivíduos dos três tipos de face; as funções de mastigação e respiração influenciam de forma importante o crescimento vertical da face; o crescimento dentoalveolar é maior na face longa que na curta ${ }^{(16)}$.

Em 2003 participaram de um estudo 15 indivíduos do sexo feminino, cinco de cada tipo de face, com idades entre 20 e 30 anos, com e sem mal oclusão. O objetivo foi avaliar a função do músculo masseter nos três tipos faciais. Observouse a influência do tipo facial na atividade eletromiográfica do músculo masseter. Sua função foi significativamente menor nos indivíduos de face longa quando comparados aos de face curta e média. Além disso, não houve diferença significativa entre as pessoas de face curta e média ${ }^{(17)}$.
Três pesquisas foram encontradas sobre tipologia facial em 2005. Em uma delas, participaram 40 indivíduos com mal oclusão do tipo Classe III de Angle esquelética; deles, 25 dolicocefálicos e 15 mesocefálicos. Teve como objetivo investigar se o tipo facial acarreta em implicações nas funções estomatognáticas. Como resultados, nos dolicocefálicos foram encontrados postura alterada de lábios, respiração oral ou oronasal, fala e deglutição com anteriorização de língua, mastigação alterada e postura de língua no assoalho durante o repouso; e nos mesocefálicos, interposição de língua na deglutição, mastigação também alterada e língua no assoalho na posição de repouso ${ }^{(8)}$.

Fizeram parte da outra pesquisa 66 sujeitos de 12 a 30 anos, no qual um dos objetivos foi verificar as proporções faciais que podiam diferenciar e caracterizar os tipos faciais no sentido vertical por meio de traçado cefalométrico. Dentre os resultados obtidos foram observadas as seguintes características: sujeitos dolicofaciais com altura facial anterior, distância dentoalveolar anterior e terço inferior da face aumentados, além de mandíbula hiperdivergente; mesofaciais com altura facial posterior, distância dentoalveolar anterior e altura facial anterior com valores equilibrados; e braquifaciais com altura facial posterior aumentada e mandíbula hipodivergente ${ }^{(9)}$.

$\mathrm{O}$ terceiro estudo teve como objetivo determinar as características cefalométricas do individuo portador de face longa ao analisar 73 telerradiografias em norma lateral. Dentre os resultados, verificou-se que os portadores de face longa apresentaram padrão de crescimento vertical, aumento da altura facial anterior total e inferior, retrognatismo maxilar e mandibular ${ }^{(23)}$.

No ano de 2006, foram duas pesquisas. Uma delas teve o intuito de comparar as medidas do lábio superior e do filtro nos tipos de face média e longa e mal oclusões dos tipos Classe I ou II de Angle. Participaram 123 crianças brasileiras leucodermas, 56 meninos e 67 meninas, com idades entre sete e onze anos. Concluiu-se que o tipo de face não interfere no comprimento do lábio superior e do filtro. Os autores discorrem que o lábio não é encurtado na face longa, ocorre na verdade um aumento da dimensão vertical da maxila ${ }^{(18)}$.

Da outra pesquisa, participaram 271 sujeitos, 168 mulheres e 103 homens, entre 14 e 44 anos e teve como objetivo verificar se há relação entre tipos faciais e presença de diastemas que se referem ao espaçamento ou ausência de contato entre dentes ligados um ao outro. Consistiu na análise de prontuários dos tratamentos ortodônticos aos quais os indivíduos haviam sido submetidos. Como resultado, os padrões mesofacial e braquifacial apresentaram mais diastemas na arcada inferior em relação ao dolicofacial, na faixa etária de 14 a $34 \operatorname{anos}^{(10)}$.

Cinco artigos foram publicados em 2007 relacionados às características dos tipos faciais. Um estudo contou com a participação de 119 adolescentes de ambos os gêneros, com idades entre 15 e 18 anos, e objetivou verificar a existência da relação entre respiração oral e tipo facial. Como resultado, não foi possível comprovar essa relação, pois não foi encontrada diferença estatisticamente significativa nessa comparação. Mas discutiu-se a idéia de que, por si só, a respiração oral não é necessariamente prejudicial para o crescimento craniofacial $^{(24)}$.

Outra pesquisa também relacionou tipo facial e respiração 
oral no mesmo ano, mas neste caso com o objetivo de investigar a relação entre tipo facial e oclusão dentária de 40 adolescentes respiradores orais de ambos os sexos, com idades entre 13 e 16 anos. Diante dos resultados, não foi possível estabelecer uma relação entre respiração oral, tipo facial e oclusão dentária ${ }^{(11)}$.

As outras três publicações referem-se a um mesmo estudo realizado em três etapas com a participação de 300 brasileiros leucodermas, 131 homens e 169 mulheres, idades entre 10 e 14 anos, de diferentes tipos faciais e mal oclusões. Os estudos tiveram como objetivo básico avaliar o crescimento médio anual da base craniana nos diferentes tipos de face e relações maxilomandibulares, pois a base craniana influencia diretamente a posição da maxila e da mandíbula. Constatouse que os valores relacionados à base craniana posterior e anterior mantêm suas proporções durante o crescimento e podem ser relacionados à forma de crescimento da maxila e da mandíbula. Por esse motivo, os dados utilizados no estudo podem ser utilizados de forma confiável para antecipar qual tipo de crescimento um paciente poderá apresentar. Isso traz informações tanto para o prognóstico, como também para o planejamento do tratamento ortopédico/ortodôntico a ser empregado e dos resultados, favoráveis ou não, esperados diante de cada tipo de face e má oclusão( ${ }^{(19,25-26)}$.

Em 2008, quatro estudos foram encontrados. Uma pesquisa foi realizada com a amostra de 88 brasileiros leucodermas, descendentes de portugueses, espanhóis e italianos, de sete a 13 anos. Teve como um dos objetivos correlacionar a base craniana e o tipo facial. Um dos resultados encontrados foi a significância entre a variável representativa do ângulo da base do crânio com o tipo facial. Ou seja, foi possível averiguar que, quanto maior o ângulo da base craniana, maior a tendência do indivíduo ser braquifacial; e quanto menor o valor desse ângulo, maior a tendência da pessoa ser dolicofacial ${ }^{(12)}$.

O outro estudo foi realizado na Turquia e teve como objetivo determinar a prevalência dos tipos faciais em adultos jovens, além de comparar medidas faciais antropométricas verticais e horizontais, segundo sexo e tipo facial. A amostra constou de 173 indivíduos, 90 homens e 83 mulheres, com idades entre 17 e 25 anos. Dentre os resultados, foi detectado dimorfismo sexual em todos os tipos faciais, principalmente nos indivíduos euriprosopos. No geral, foram observados: larguras facial e nasal menores e alturas faciais superior e total maiores nos leptoprosopos e maior largura facial horizontal nos euriprosopos ${ }^{(13)}$.

A terceira pesquisa encontrada objetivou verificar se há relação entre o tamanho do espaço aéreo nasofaríngeo e bucofaríngeo e os tipos faciais em pessoas sem distúrbio funcional respiratório, sem tratamento ortodôntico ou ausências dentárias. Participaram 90 pessoas, 55 homens e 35 mulheres, de nove a 16 anos. Não foram encontradas diferenças estatisticamente significativas entre os tamanhos dos espaços nasofaríngeo e bucofaríngeo ao serem relacionados aos três tipos de face ou aos dois gêneros ${ }^{(27)}$.

A última referência desse ano refere-se à pesquisa em que participaram 105 adultos leucodermas, 34 homens e 71 mulheres, de 20 a 40 anos. Objetivou comparar duas formas de classificação do tipo de face: a partir da cefalometria e da análise facial clínica por meio da observação de fotografias, além de descrever os valores de sete medidas antropométricas faciais diretas, três índices e três proporções faciais, segundo tipo facial e gênero, na tentativa de se determinar o tipo de face apenas a partir da antropometria. Como resultados, observou-se que a classificação da face apenas a partir da análise facial não foi considerada confiável se utilizada isoladamente. Além disso, os tipos faciais classificados por meio da cefalometria apresentaram diferenças estatisticamente significativas para quatro medidas antropométricas faciais, três índices e uma proporção faciais do gênero masculino, e cinco medidas e uma proporção do feminino. Houve dimorfismo de gênero, com valores maiores das medidas para o gênero masculino. Os valores da altura facial anterior, da face média e da face inferior foram maiores para os indivíduos dolicofaciais, intermediários para os mesofaciais e menores para os braquifaciais. Ordem de valores inversa foi encontrada para a medida da altura facial posterior. Os índices e proporções antropométricas faciais promoveram dados confiáveis para a determinação das faces: dolicofacial em homens; dolicofacial e braquifacial em mulheres ${ }^{(28)}$.

Por fim, no ano de 2009, um estudo foi realizado com o objetivo de comparar a atividade eletromiográfica dos músculos masseter e a porção anterior do temporal em indivíduos com diferentes tipos faciais. Participaram 44 sujeitos, com idades entre 18 e 35 anos, avaliados em posição de repouso e máxima intercuspidação dentária. Como resultado, não foram encontradas diferenças estatisticamente significativas com relação à atividade eletromiográfica da musculatura avaliada ao serem comparados os três tipos de face ${ }^{(14)}$.

\section{DISCUSSÃO}

A partir da revisão foi observada divergência no que diz respeito às várias terminologias utilizadas para classificação dos tipos faciais. De forma imprecisa, podem-se associar os termos: face longa ${ }^{(4-5,16-18,23,29)}$ a tipo dolicofacial, dolicocefálico, leptoprosopo ${ }^{(1-14,30-31)}$, hiperdivergente ${ }^{(9,15)}$, perfil retrognata, retrovertido ou convexo ${ }^{(2,19)}$; face média ${ }^{(4-5,16,18)}$ a tipo mesofacial, mesocefálico, mesoprosopo ${ }^{(1-14,30-31)}$, neutro ${ }^{(9,15)}$, perfil ortognata, neutrovertido ou reto ${ }^{(2,19)}$; e face $\operatorname{curta}^{(4-5,16,18)}$ a tipo braquifacial, braquicefálico, euriprosopo ${ }^{(1-14,30-31)}$, hipodivergente ${ }^{(9,15)}$, perfil prognata, provertido ou côncavo ${ }^{(2,19)}$. Entretanto, deve-se levar em consideração que cada terminologia carrega conceituação e características próprias, não necessariamente correspondentes.

A partir do levantamento bibliográfico realizado, foram observadas as seguintes características para o indivíduo de face longa: postura alterada de lábios, respiração oral ou oronasal, fala e deglutição com anteriorização de língua, mastigação alterada e postura de língua no assoalho durante o repouso $^{(8)}$; qualidade vocal grave com tendência a ser abafada e escura, associada à ressonância posterior ${ }^{(22)}$; maior distância dentoalveolar anterior ${ }^{(9,16)}$; maior altura facial anterior ${ }^{(9,13,23,28)}$; maior altura facial anterior inferior ${ }^{(9,23,28)}$; alturas da face média e maiores e facial posterior menores ${ }^{(28)}$; largura facial e nasal menores e altura facial superior maior ${ }^{(13)}$; menor atividade eletromiográfica do músculo masseter ${ }^{(17)}$; mandíbula hiperdivergente ${ }^{(9)}$; e menor ângulo da base do crânio ${ }^{(1)}$. Esses achados condizem com a literatura mais antiga ${ }^{(1-4,6)}$. 
Com relação às pessoas de face média foram encontradas: língua no assoalho na posição de repouso ${ }^{(8)}$; altura facial anterior e posterior ${ }^{(9,28)}$ e altura da face média e inferior ${ }^{(28)}$ com valores intermediários; e distância dentoalveolar anterior com valores equilibrados ${ }^{(9)}$. Esses dados vão ao encontro de referências anteriores ${ }^{(1-2)}$. Entretanto, segundo alguns autores ${ }^{(1,4)}$, as funções estomatognáticas geralmente não apresentam alterações, diferente do encontrado em uma pesquisa $^{(8)}$, ou seja, interposição de língua na deglutição, mastigação também alterada. Um achado para o qual não foram encontradas informações na literatura pesquisada sobre a maior incidência de diastemas na arcada inferior no individuo de face média ${ }^{(10)}$.

No que diz respeito às características das pessoas com face curta, os estudos relataram aspectos também encontrados em literatura mais remota ${ }^{(1-2,4,6)}$, ou seja, qualidade vocal aguda com tendência metálica e ressonância faríngea ${ }^{(22)}$; altura facial anterior, da face média e da face inferior menores ${ }^{(28)}$ e altura facial posterior maior ${ }^{(9,28)}$; maior largura facial horizontal ${ }^{(13)}$; menor crescimento dentoalveolar ${ }^{(16)}$; mandíbula hipodivergente ${ }^{(9)}$; maior ângulo da base do crânio ${ }^{(12)}$; e maior presença de diastemas na arcada inferior ${ }^{(10)}$.

Vários aspectos foram controversos ao se caracterizar os tipos de face. Alguns autores ${ }^{(14,17)}$ não encontraram diferenças estatisticamente significativas com relação à atividade eletromiográfica do músculo masseter ao serem comparados os três tipos de face. Mas essa atividade foi considerada menor nos indivíduos de face longa em um dos estudos ${ }^{(17)}$. Segundo referências mais antigas ${ }^{(1,4,6)}$, a musculatura da pessoa com face longa é, no geral, estirada e hipotônica e os músculos elevadores da mandíbula delgados; e os indivíduos com face curta apresentam musculatura forte, com músculos elevadores da mandíbula espessos, inserção ampla em seu corpo, em especial o músculo masseter que se apresenta encurtado e hipertônico. Mais um ponto a ser considerado é que, segundo dois estudos ${ }^{(8,16)}$, há relação entre mastigação e forma de crescimento facial, consequentemente com o tipo facial.

Outro aspecto contraditório foi relacionado à associação entre a respiração e o tipo facial. Alguns autores afirmam, a partir de suas pesquisas ${ }^{(11,24)}$, que não é possível estabelecer uma relação entre modo respiratório e tipo facial, dado que não foi descrito na literatura pesquisada ${ }^{(1,3-4)}$ a qual caracteriza o dolicofacial por apresentar frequentemente respiração oral e o braquifacial, nasal. Um desses estudos ${ }^{(24)}$ discutiu também a idéia de que, por si só, a respiração oral pode não ser necessariamente prejudicial para o crescimento craniofacial, mas alguns autores ${ }^{(8,16)}$ não concordam com esse ponto de vista. Além disso, de acordo com outro estudo ${ }^{(27)}$ não há relação entre os tamanhos dos espaços nasofaríngeo e bucofaríngeo e os três tipos de face, mas segundo alguns autores ${ }^{(1,3-4)}$ as vias aéreas superiores do indivíduo de face longa tendem a ser mais estreitas e alongadas e na face curta, mais largas e curtas.

Mais um ponto que gerou controvérsia foi a associação entre o comprimento do lábio superior e do filtro e o tipo de face. Uma pesquisa ${ }^{(18)}$ concluiu que não há relação entre esses aspectos e que, na face longa, o lábio superior não é encurtado como referido por outros autores ${ }^{(1,3-4,6,620)}$, o que ocorre é um aumento da dimensão vertical da maxila.
Dessa forma, pôde-se perceber que cada tipo facial apresenta características estéticas, ósseas, musculares e funcionais próprias ${ }^{(1)}$. Todavia deve-se ter em mente que nem sempre todas as características poderão estar presentes. Isso se deve ao fato de cada pessoa ser única e poder apresentar diferentes compensações, que dependem do grau da mal oclusão e do padrão de crescimento craniofacial.

Outro aspecto que deve ser levado em consideração no que diz respeito às características faciais é que se deve prestar atenção à população pesquisada e apresentada nos estudos. Isso porque foi observado, por exemplo, que a população brasileira apresenta a face menos protruída, perfil mais côncavo e menor proeminência do queixo que os norte-americanos ${ }^{(32)}$.

Além disso, deve-se ressaltar que os tipos faciais não precisam ser taxados como bons ou ruins, mediante as características por eles apresentadas. Na verdade, eles devem ser considerados como favoráveis ou desfavoráveis para o tratamento a ser realizado ${ }^{(7)}$. O ideal é também considerar, além das características dos tipos faciais, se a face é considerada agradável, aceitável ou desagradável ${ }^{(21,33-36)}$, e buscar sempre a melhor estética facial e a função mais adequada para se alcançar resultados mais satisfatórios dos tratamentos realizados, também do ponto de vista do profissional, mas principalmente para o paciente e seus familiares.

\section{COMENTÁRIOS FINAIS}

Diante da revisão, alguns aspectos foram controversos ao se comparar os tipos faciais como a atividade eletromiográfica do músculo masseter, o modo respiratório e o comprimento do lábio superior e do filtro. Além disso, foi observada divergência quanto às terminologias adotadas para sua classificação.

Outro ponto a ser comentado é que os parâmetros de normalidade encontrados na literatura podem não representar a realidade brasileira em conseqüência da população ser bastante miscigenada, com grande variabilidade das características apresentadas. Por essa razão, o profissional que atua com a face, seja o fonoaudiólogo ou o ortodontista, deve ter em mente essa questão na atuação clínica e na seleção dos sujeitos para pesquisas. Vale ressaltar que é fundamental a realização de mais pesquisas de campo que verifiquem se as características dos tipos faciais encontradas, principalmente em referências mais antigas, são realmente aplicáveis à população brasileira.

Pôde-se constatar que conhecer o tipo facial e correlacionálo às funções estomatognáticas, musculatura e oclusão é um fator importante para a prática clínica, mas o profissional deve ser flexível ao comparar as características do paciente da clínica com a literatura. Dessa forma, pode-se evitar determinar uma anormalidade ou atipia, ou mesmo generalizações inadequadas, caso estejamos diante de uma variabilidade localizada ou de uma adaptação.

\section{AGRADECIMENTOS}

À Coordenação de Aperfeiçoamento de Pessoal de Nível Superior (CAPES), pelo auxílio financeiro e pelo incentivo à pesquisa. 


\begin{abstract}
The human face, with its bone and muscular structures, present singular and peculiar characteristics. It may be classified in three basic types, which are related with shape variation and craniofacial configuration, both vertically and horizontally, influencing directly the dental occlusion, facial harmony, orofacial muscles and stomatognathic functions. For this reason, diagnosing facial type is important to speech-language pathology practice. The aim of the present study was to perform a literature review regarding facial type characteristics, presenting recent researches and studies about the theme. A bibliographical research was carried out in the databases LILACS, SciELO, Web of Science and Google Scholar, in addition to books, dissertations and thesis about the subject in the past ten years. Several field researches proved some characteristics of facial types found, especially, in the older references. Some aspects, however, were controversial when the facial types were compared, such as the eletromyographic activity of the masseter muscle, respiratory mode and the length of the upper lip and of the philtrum. It was possible to conclude that knowing the facial type and correlating it to the stomatognathic functions, muscles and occlusion is an important factor for practical clinic, but the professional should be flexible when comparing the pacient's characteristics with results found in the literature. Thus, it is possible to avoid determining an abnormality or atypia when the case is only a variability or adaptation.
\end{abstract}

Keywords: Face/physiology; Stomatognathic system; Dental occlusion; Diagnosis

\title{
REFERÊNCIAS
}

1. Bianchini EM. A cefalometria nas alterações miofuncionais orais- diagnóstico e tratamento fonoaudiológico. 5a ed rev. e ampl. Carapicuíba: Pró-Fono; 2002.

2. Enlow DH, Poston WR, Bakor SF. Crescimento facial. 3a ed. São Paulo: Artes Médicas; 1993.

3. Marchesan IQ. O trabalho fonoaudiológico nas alterações do sistema estomatognático. In: Marchesan IQ, Bolaffi C, Gomes IC, Zorzi JL, organizadores. Tópicos em fonoaudiologia. São Paulo: Lovise; 1994. p.83-96.

4. Krakauer LH. Alteração de funções orais nos diversos tipos faciais. In: Marchesan IQ, Bolaffi C, Zorzi JL, Gomes IC, organizadores. Tópicos em fonoaudiologia 1995. São Paulo: Lovise; 1995. p.147-54.

5. Dragone ML, Coleta RD, Bianchini EM. Encaminhamentos fonoaudiológicos e ortodônticos: concordâncias. In: Marchesan IQ, Zorzi JL, Gomes IC, organizadores. Tópicos em fonoaudiologia 1997/1998. São Paulo: Lovise; 1998. p.307-27.

6. Felício CM. Fonoaudiologia aplicada a casos odontológicos: motricidade oral e audiologia. São Paulo: Pancast; 1999. Problemas ortodônticos e o papel das (dis)funções orofaciais; p.171-96.

7. Cometti JC. Biotipo facial em odontologia. Prensa Méd Argent. 1999;86(8):819-22.

8. Pereira AC, Jorge TM, Ribeiro Júnior PD, Berretin-Felix G. Características das funções orais de indivíduos com má oclusão classe III e diferentes faciais. Rev Dental Press Ortodon Ortopedi Facial. 2005;10(6):111-9.

9. Santos SH, Moraes LC, Medici Filho E, Castilho JC, Moraes ME. Aplicação do método linear e geométrico utilizando radiografias cefalométricas laterais, para diferenciar e identificar a proporção divina em três tipos faciais. Ciênc Odontol Bras. 2005;8(3):10-21.

10. Canuto MS, Assis RS, Gouveia PM, Nemr K. Análise comparativa entre presença de diastemas e tipos faciais. Rev CEFAC. 2006;8(2):162-70.

11. Sies ML, Farias SR, Vieira MM. Respiração oral: relação entre o tipo facial e a oclusão dentária em adolescentes. Rev Soc Bras Fonoaudiol. 2007;12(3):191-8.

12. Araújo MC, Nahás AC, Cotrim-Ferreira FA, Carvalho PE. Estudo cefalométrico da correlação da anatomia da base craniana com o padrão facial e as bases apicais. Rev Dent Press Ortodon Ortopedi Facial. 2008;13(4):67-76

13. Arslan SG, Genç C, Odabaş B, Kama JD. Comparison of facial proportions and anthropometric norms among Turkish young adults with different face types. Aesthetic Plast Surg. 2008;32(2):234-42.

14. Vianna-Lara MS, Caria PH, Tosello Dde O, Lara F, Amorim MM. Electromyographic activity of masseter and temporal muscles with different facial types. Angle Orthod. 2009;79(3):515-20.
15. Zielinsky L. Crecimiento, desarollo y maduración. Parte II. Rev Cubana Ortod. 1997;12(1):46-63.

16. Dalmagro Filho L, Maria FT, Souza RS, Takahashi R, Takahashi T, Rino W. Dimensão vertical da face: revisão de literatura. Arq Ciências Saúde UNIPAR. 2002;6(2):187-91.

17. Rodrigues KA, Rahal A. A influência da tipologia facial na atividade eletromiográfica do músculo masseter durante o apertamento dental em máxima intercuspidação. Rev CEFAC. 2003;5:127-30.

18. Daenecke S, Bianchini EM, Silva AP. Medidas antropométricas de comprimento de lábio superior e filtro. Pró-Fono. 2006;18(3):249-58.

19. Pieri LV, Faltin Júnior K, Ortolani CL, Faltin RM, Almeida MA. Crescimento da base craniana nos diferentes tipos faciais nos relacionamento maxilomandibulares ortopédicos de classe I, II e III Parte 1. Rev Dent Press Ortodon Ortopedi Facial. 2007;12(1):110-7.

20. Bianchini EM. Avaliação fonoaudiológica da motricidade oral: distúrbios miofuncionais orofaciais ou situações adaptativas. Rev Dental Press Ortodon Ortopedi Facial. 2001;6(3):73-82.

21. Capelozza Filho L. Diagnóstico em ortodontia. Maringá: Dental Press; 2004. 512p.

22. Oliveira VL, Pinho SM. A qualidade da voz e o trato vocal nos indivíduos de face curta e face longa. In: Pinho SM. Tópicos em voz. Rio de Janeiro: Guanabara Koogan; 2001. p.81-8.

23. Cardoso MA, Bertoz FA, Capelozza Filho L, Reis SA. Características cefalométricas do padrão face longa. Rev Dental Press Ortodon Ortopedi Facial. 2005;10(2):29-43.

24. Bianchini AP, Guedes ZC, Vieira MM. Estudo da relação entre a respiração oral e o tipo facial. Rev Bras Otorrinolaringol. 2007;73(4):500-5.

25. Pieri LV, Faltin Júnior K, Ortolani CL, Faltin RM, Almeida MA. Crescimento da base craniana nos diferentes tipos faciais nos relacionamento maxilomandibulares ortopédicos de classe I, II e III Parte 2 (Crescimento médio de Ba-Na, CC-Na e CC-Ba). Rev Dental Press Ortodon Ortopedi Facial. 2007;12(2):71-87.

26. Pieri LV, Faltin Júnior K, Ortolani CL, Faltin RM, Almeida MA. Crescimento da base craniana nos diferentes tipos faciais nos relacionamento maxilomandibulares ortopédicos de classe I, II e III - Parte 3 (Crescimento médio de CF-Po). Rev Dental Press Ortodon Ortopedi Facial. 2007;12(3):126-35.

27. Castro AM, Vasconcelos MH. Avaliação da influência do tipo facial nos tamanhos dos espaços aéreos nasofaríngeo e bucofaríngeo. Rev Dental Press Ortodon Ortopedi Facial. 2008;13(6):43-50.

28. Ramires RR. Correlação entre cefalometria e antropometria para determinação do tipo facial [dissertação]. São Paulo: Pontifícia Universidade Católica de São Paulo; 2008. 
29. Capelozza Filho L, Cardoso MA, Li An T, Bertoz FA. Características cefalométricas do padrão face longa: considerando o dimorfismo sexual. Rev Dental Press Ortodon Ortopedi Facial. 2007;12(2):49-60.

30. Silva Filho OG, Queiroz AP, Herkrath FJ, Silva GF. Correlação entre padrão facial e relação sagital entre os arcos dentários no estágio de dentadura decídua: considerações epidemiológicas. Rev Dental Press Ortodon Ortopedi Facial. 2008;13(1):101-12.

31. Silva Filho OG, Herkrath FJ, Queiroz AP, Aiello CA. Padrão facial na dentadura decídua: estudo epidemiológico. Rev Dental Press Ortodon Ortopedi Facial. 2008;13(4):45-59.

32. Sant'Ana E, Kuriki EU, Arnett W, Lautenschläger GA, Yaedu RY. Avaliação comparativa do padrão de normalidade do perfil facial em pacientes brasileiros leucodermas e em norte-americanos. Rev Dental Press Ortodon Ortopedi Facial. 2009;14(1):80-9.
33. Reis SA, Capelozza Filho L, Cardoso MA, Scanavini MA. Características cefalométricas dos indivíduos Padrão I. Rev Dental Press Ortodon Ortopedi Facial. 2005;10(1):67-78.

34. Edler R, Agarwal P, Wertheim D, Greenhill D. The use of anthropometric indices in the measurement of facial attractiveness. European J Orthod. 2006;28(3):274-81.

35. Reis SA, Abrão J, Capelozza Filho L, Claro CA. Análise facial subjetiva. Rev Dental Press Ortodon Ortopedi Facial. 2006;11(5):159-72.

36. Trevisan F, Gil CT. Análise fotogramétrica e subjetiva do perfil facial de indivíduos com oclusão normal. Rev Dental Press Ortodon Ortopedi Facial. 2006;11(4):24-35. 